\title{
4Pi-Confocal Microscopy Provides Three-Dimensional Images of the Microtubule Network with 100- to 150-nm Resolution
}

\author{
Matthias Nagorni and Stefan W. Hell \\ High Resolution Optical Microscopy Group, Max-Planck-Institutefor Biophysical Chemistry, D-37070 Göttingen, Germany
}

Received May 30, 1998; and in revised form September 3, 1998

\begin{abstract}
We show the applicability of 4Pi-confocal microscopy to three-dimensional imaging of the microtubule network in a fixed mouse fibroblast cell. Comparison with two-photon confocal resolution reveals a fourfold better axial resolution in the 4Pi-confocal case. By combining 4Pi-confocal microscopy with Richardson-Lucy image restoration a further resolution increase is achieved. Featuring a threedimensional resolution in the range $100-150 \mathrm{~nm}$, the 4Pi-confocal (restored) images are intrinsically more detailed than their confocal counterparts. Our images constitute what to our knowledge are the best-resolved three-dimensional images of entangled cellular microtubules obtained with light to date.
\end{abstract}

\section{INTRODUCTION}

Owing totheir three-dimensional imaging capability, confocal fluorescence microscopes enjoy great popularity for structural and functional imaging in biology; for a review see the volume by Pawley (1995) and the references therein. However, the resolution of confocal microscopes, and also that of two-photon microscopes (Denk et al., 1990), is limited to about 200 and $500-800 \mathrm{~nm}$ in the lateral and axial directions, respectively. Lateral resolution of the order of $80 \mathrm{~nm}$ has been shown by near-field fluorescence microscopy of biological specimens (Hulst et al., 1997). However, relying on the interaction between a tip and a surface, near-fiel d optical microscopy develops its potential only at the specimen surface. Hence, far-field microscopes such as the confocal and multiphoton will continue to play an important role in biological imaging in the future.

4Pi-confocal microscopy (Hell, 1990) is a concept to improve the spatial resolution in the far-fiel d fluorescence microscopy. With a lateral resolution of $\sim 200$ $220 \mathrm{~nm}$ and an axial resolution of $\sim 140 \mathrm{~nm}$, the (two-photon excitation) 4Pi-confocal fluorescence microscope (Hell and Stelzer, 1992a) improves the axial resolution by a factor of $\sim 3-4$ over that of a standard confocal or multiphoton microscope (Schrader and Hell, 1996). The axial resolution of a 4Pi-confocal microscope is better than its lateral counterpart. This stems from the operational principle of the $4 \mathrm{Pi}$-confocal microscope which is the improvement of the total aperture with two opposing high aperture lenses.

When a specimen point is coherently illuminated through opposing lenses (4Pi of type A), the two focused, spherical wavefronts add up to form a single wavefront (Hell and Stelzer, 1992b). For constructive interference of the wavefronts, they produce a focus (point-spread function, PSF) with a pronounced main maximum, as well as an axial lobe above and be neath the focal plane. The 4-times-narrower main maximum resolves better, but, if no other measures are taken, the sidel obes introduce ghost images. To suppress the lobes, the fluorophore is usually excited by near-infrared two-photon absorption, and the fluorescence is collected through a confocal pinhole (Hell and Stelzer, 1992a). Subsequent fast-linedeconvolution carried out during image acquisition re moves the lobes entirely (Hänninen et al., 1995).

A common requirement of most imaging devices is that their imaging properties, i.e. the PSF, are invariant in space (Goodman, 1968). The necessity of deconvolution in 4Pi-confocal microscopy makes space-invariance of the PSF of paramount importance. Basically, two requirements have to be met to guarantee space invariance in a $4 \mathrm{Pi}$-confocal image. First, the foci of the opposing lenses need to overlap with high accuracy. Second, the relative phase between the two interfering spherical wavefronts must be constant. During earlier stages of development, it was doubted that these requirements could be met in a cell (Lindek et al., 1994). H owever, initial investigations with actin fibres of a mouse fibroblast cell have shown that the relative phase of the interfering wavefronts can be kept constant over rather large volumes in the cytoplasm (Egner et al., 1998; Hell et al., 1997; Schrader et al., 1998b). Moreover, twophoton excitation 4Pi-confocal microscopy combined 
with image restoration provided 3D images of the actin filaments with an unsurpassed 3D resolution in the 100-nm range (Hell et al., 1997).

The 3D imaging of the microtubule network is particularly challenging because the microtubules appear as a densely arranged network of ultrathin (24 nm) fibres (Darnell et al., 1990). It is therefore interesting to investigate the potential of $4 \mathrm{Pi}$ confocal microscopy for this application. In this paper, after briefly recapitulating the operational principles of $4 \mathrm{Pi}$-confocal microscopy, we show first 3D 4Pi-confocal images of the microtubule network of a mouse fibroblast cell. We find that the 4Piconfocal microscope delivers images with far greater detail than its confocal counterpart.

\section{4PI-CONFOCAL MICROSCOPY}

We used a two-photon excitation 4Pi-confocal microscope of type A as described in Hänninen et al. (1995), Hell (1997), Hell et al. (1994) and Schrader and Hell (1996). Two-photon excitation of the fluorophore was accomplished with a pulsed Ti:Sa laser (Mira $900 \mathrm{~F}$, Coherent) operating at a wavel ength of $780 \mathrm{~nm}$. The back apertures of the opposing objective lenses were illuminated with $\sim 0.5 \mathrm{~mW}$, which rendered about 250-350 $\mu \mathrm{W}$ of time-averaged power in the focal region. We used oil immersion lenses with a numerical aperture of 1.4 (Leica PL Apo 100×); the lenses were not preselected by the manufacturer. Confocal and multiphoton microscopes usually scan the beam in the focal plane $(x, y)$ and stepwise translate the object along the optical axis (z) with a typical pixel dwell time of 2-100 $\mu \mathrm{s}$. We chose slower, but more precise stage scanning, whereby the sample is scanned with a piezo stage (Melles Griot, Cambridge, England) through the common focus of the two lenses. The typical dwell time was $2 \mathrm{~ms}$ per pixel. In order to benefit from the improved axial resolution our images also featured a denser pixelation along $z$, of typically $30 \mathrm{~nm} /$ pixel. The pixelation along $x$ and y was typically $50-120 \mathrm{~nm}$. Our fast-scan axis was the Z-axis, so that our images were initially axial images, i.e., $x z$ images. By displacing the sample al ong the $Y$-axis, slice by slice, we obtained a 3D data stack that can be visualised from all directions, just as any other confocal or multiphoton 3D data stack.

Fluorescence light was filtered through a shortpass dichroic mirror and 2-mm-thick coloured glass (BG39, Schott). The fluorescence was detected with an avalanche photodiode photon-counting Module (SPCM-121; EG\&G, Canada) featuring a high sensitivity in the visible range (Kano et al ., 1996). The foci of the two lenses are kept tight by controlling one of the lenses with a piezo stage operating with 10-nm precision. The transmitted excitation light passing both lenses was directed on a low-cost CCD camera which allowed us to view any small axial and lateral displacements of the interfering foci. The diffraction image of the transmitted beam was taken as a reference for the alignment and stability of the setup.

To avoid phase changes induced by air circulation we covered the $4 \mathrm{Pi}$-confocal microscope with a cardboard box. The box featured flexible openings in order to allow the exchange of the specimens and also the use of an eyepiece for viewing the sample in a conventional epifluorescence mode. The laboratory room was temperature regulated to $21-22^{\circ} \mathrm{C}$, but no further measures weretaken to stabilise the environment.

In order to control the relative phase of the focused wavefronts, we used a piezo-driven mirror. This deviceall owed us to adjust the relative optical length of the two paths of the excitation beams with a precision of $\lambda / 40$. As the mounting medium of our specimens (glycerol) had a refractive index smaller than that of immersion oil and glass, $\mathrm{n} \approx 1.47$ as opposed to $n=1.51$, the optical paths' lengths and hence the phase of the two beams changed during the axial translation of the sample. It has been shown that this phase change is a linear function of the scan and can beactively compensated by synchronously moving the piezo mirror with the axial movement of the sample (Egner et al., 1998; Hell et al., 1997). Therefore, in a largely uniform volume such as the cytoplasm of a fixed cell, the 4Pi PSF can be kept constant even in the presence of a significant refractive index mismatch between the mounting medium and the glass cover slip.

The PSF was determined by recording images of fluorescent beads. The beads were mounted in glycerol in order to mimic the optical situation encountered in the sample. We recorded a stack of 20 parallel $x z$ images that were $45 \mathrm{~nm}$ apart in the $\mathrm{y}$ direction. The pixel sizes in the slices were $28 \mathrm{~nm}$ in lateral $(\mathrm{x})$ and $18 \mathrm{~nm}$ in axial $(\mathrm{z})$ direction. Each slice had a dimension of $128 \times 32$ pixels. The maximum number of counts per pixel was 575 in the brightest region.

\section{IMAGE DECONVOLUTION AND RESTORATION IN 4PI-CONFOCAL MICROSCOPY}

We used two different approaches to eliminate the effect of the two sidelobes of the 4Pi-confocal PSF. The first approach was a linear, one-dimensional (point) deconvolution that relied only on the position and height of the lobes (Hänninen et al., 1995; Schrader and Hell, 1996). Its mathematical simplicity allowed this method to be carried out on-line, i.e., during data acquisition. For full three-dimensional restoration we used the iterative Richardson-Lucy 
image restoration method (Lucy, 1974; Richardson, 1972), which was performed after data acquisition.

Although both methods are referred to as "deconvolution," they are fundamentally different. The online point deconvolution is a true mathematical deconvolution. It is a linear operation acting as a linear spatial filter that is carried out line by line only along the optic axis. The effect of the point deconvolution is to remove the "ghost images" induced by the lobes, but it leaves the shape of the central maximum essentially untouched. The point deconvolution does not enlarge the bandwidth of the optical transfer function (Gu and Sheppard, 1994) of the 4Pi-confocal microscope. It uses merely the information of the location and the relative height of the lobes and not that of the full PSF. It must not be confused with common image restoration methods (Carrington et al., 1995; Holmes et al., 1995; Van der Voort and Strasters, 1995), which are in fact iterative approaches rather than deconvolutions. Point deconvolution does not require any a priori information about the object.

The principles of point deconvolution can be explained as follows. As the object is scanned in the axial direction, each axial scanline exhibits the typical $4 \mathrm{Pi}$ response in the same manner. Onedimensional deconvolution requires the relative height of the sidelobes to be unchanged along any axial scanline. This allows us to deconvolve each scanline separately. This requirement is met since as a good approximation, in two photon 4Pi-confocal microscopy, the total PSF $h(x, y, z)$ can be decomposed into an axial function $h_{1}(z)$ of a single line scan and a factor $c(x, y)$, which is constant for any given axial scanline through $(x, y)$. The reason is that the nonlinearity induced by two-photon excitation and confocal detection suppresses the outer parts of the focus, otherwise characterised by ringing. Hence, we can write,

$$
h(x, y, z) \cong c(x, y) h_{l}(z)
$$

$h_{1}(z)$ is the convolution of a peak function $h_{\text {peak }}(z)$, which describes the shape of one peak, and the lobe function I (z), which contains information about the position and relative height of the lobes

$$
h_{l}(z) \cong h_{\text {pøak }}(z) \otimes I(z) \text {. }
$$

Direct algebraic inversion of I ( $z$ ) leads to a Toeplitz problem, which can be solved numerically. As a result we obtain an inverse filter in the spatial domain, here defined as $\mathrm{I}^{-1}(\mathrm{z})$. This filter unambiguously eliminates the sidelobes of the 4Pi PSF and leaves an effective PSF of $c(x, y) h_{\text {peak }}(z)$.

Poisson shot noise of the fluorescence and small instabilities in the shape of the interference pattern cause the point deconvolution to also produce negative values. We handled this by setting negative values to zero, which is acceptable as long as the noise and the fluctuations are below $5 \%$. To reduce Poisson noise, the image was filtered with a $3 \times 3 \times 3$ weighted mean filter after deconvolution. As we will show in the next section, 4Pi-confocal microscopy with fast point deconvolution is sufficient to produce better-resolved images of the microtubule network.

In the past few years, large progress has been made in the field of image restoration (Carrington et al., 1995; Van Kempen et al., 1997; Verveer and J ovin, 1998). Furthermore, it has been shown that iterative image restoration can be successfully combined with 4Pi-confocal microscopy, resulting in an even higher 3D resolution (Hell et al., 1997). So far, restoration through Maximum Likelihood Estimation (MLE) has been successfully applied. There is a scientific discussion about which restoration method is optimal, which is a subject of active research itself. By selecting a particular iterative image restoration we do not intend to imply the general superiority of this method but only follow two lines. First, image restoration is a good test for the translation invariance of the $4 \mathrm{Pi}$-confocal PSF in the 3D image. Second, the combination of high quality $4 \mathrm{Pi}$-confocal $3 \mathrm{D}$ images with restoration should result in a further increase in 3D resolution in far-field microscopy.

Of the various approaches and algorithms we chose the Richardson-Lucy (RL) al gorithm, a method which is widely used in astronomy. This classical algorithm is readily implemented in a computer program and can be shown to converge towards the solution provided by the MLE technique (Lucy, 1974). Many other methods need a more explicit model for noise, which in the case of 4Pi microscopy is not straightforward to implement. In addition to Poisson shot noise, 4Pi images also contain some noise caused by small fluctuations in the relative height of thesidel obes. This "phase noise" must al so be considered. Since small sidelobe fluctuations might be specimen induced, statistical modelling is not necessarily appropriate. The RL algorithm has the disadvantage of being slow in comparison with other methods, but, so far, computation time has not played an important role for our restoration application, since the point deconvolution provides a fast option al ready.

The $\mathrm{RL}$ restoration algorithm requires the precise knowledge of the complete 3D 4Pi-confocal PSF. The latter was established by recording a 3D 4Pi-confocal image of a 121-nm fluorescent bead (excitation 520/ emission $580 \mathrm{~nm}$; Molecular Probes, Oregon). For this purpose, the beads were dried on a standard coverslip and mounted with glycerol using a second 
coverslip. As the diameter of the bead is finite, the $4 \mathrm{Pi}$-confocal 3D image of the bead is the convolution of the 4Pi-confocal PSF with the bead object function. This fact must be taken into account when restoring the $3 \mathrm{D}$ image.

The $\mathrm{RL}$ restoration was done in the following steps. First, we resampled the stack on a finer grid and matched it to the sampling of the bead PSF. Next, we took into account that the measured bead PSF is actually a 3D convolution of a (121-nm diameter) bead with the 4Pi-confocal PSF. In principle, this can be done in two ways, either by deconvolving the measured PSF or by convolving the measured image with a band-limited sphere of appropriate diameter. We chose the latter approach. The restored stack was finally resampled to reduce the number of slices to its initial value.

\section{SPECIMENS}

We applied 4Pi-confocal microscopy to the imaging of microtubules in Balbc 3T3 mouse fibroblast cells grown on a coverslip. The microtubules werelabelled with monoclonal antibodies (21D3) to tubulin. The secondary bodies used were goat anti-mouse IgG labelled with the dye Oregon Green 488 (excitation $488 \mathrm{~nm}$, emission $520 \mathrm{~nm}$, Molecular Probes). The labelling ratio was five fluorophores per protein. The cells were fixed in $3 \%$ paraformaldehyde containing $1 \mathrm{mM}$ EGTA at $4^{\circ} \mathrm{C}$ for $15 \mathrm{~min}$. The coverslip with the fibroblasts was then mounted on a second coverslip. The mounting medium was glycerol (Merck, Darmstadt, Germany) containing 2\% dapco (Serva, Heidelberg, Germany) as anti-fading agent. An overview of the sample as obtained by a conventional confocal microscope is shown in Fig. 1.

\section{RESULTS}

In order to illustrate and quantify the improvement in resolution we always recorded pairs of images from the same site of the specimen, that is as both a confocal and a 4Pi-confocal image. In our system a high-end confocal image is obtained simply by obstructing one of the twolenses. F or the confocal image we also doubled the illumination power of the remaining objective so as to ensure similar total illumination flux for both images. The relative phase of the excitation beams was adjusted prior to acquisition so as to obtain constructive interference in the centre of the common focus. This was accomplished by observing the one-dimensional response of a single microtubule.

Figure 2a shows a confocal $x z$ slice taken in the mouse fibroblast cell. It consists of $128 \times 128$ pixels and it is part of a stack of 32 parallel $x z$ images. The pixel sizes were $49 \mathrm{~nm}$ in lateral (x) and $28 \mathrm{~nm}$ in axial (z) direction. In the direction perpendicular to

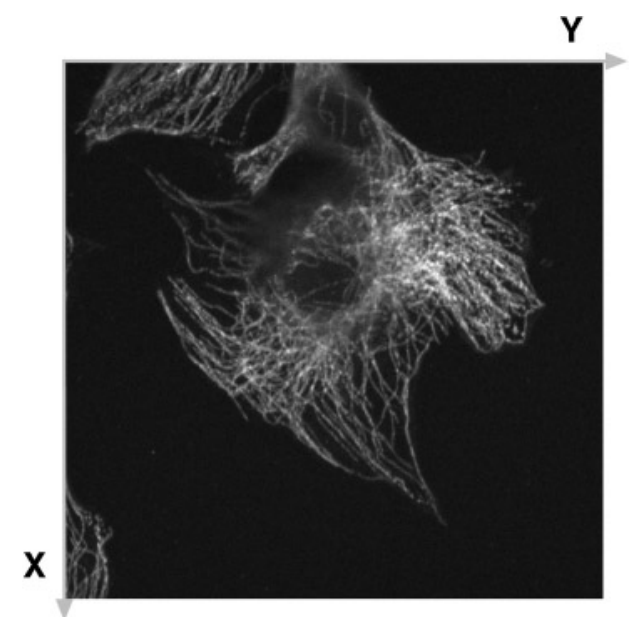

FIG. 1. Overview image of a fibroblast cell with fluorescently labelled microtubules, as used in the experiments described in Figs. 2-6. The image has been acquired in a conventional beamscanning confocal microscope (Zeiss, LSM 410).

the slice (y) the pixel step was $113 \mathrm{~nm}$. Typical acquisition time for a slice of $128 \times 128$ pixels was 82 $\mathrm{s}$. The maximum brightness in the images was of the order of 300 counts per pixel.

Poorer axial resolution can be clearly observed in the confocal image, manifested as a spread along the optic axis. Fig. 2c shows the (raw) data as obtained from the photodetector of the 4Pi-confocal microscope. The 4Pi and the confocal data stack were consecutively taken at the same site of the specimen. In the 4Pi-confocal data, the effect of the main maximum and the lobes is also readily recognised. Careful scrutinisation of the data of Fig. 2c shows that the PSF is largely constant throughout the slice. This allows us to point deconvolve and remove the axial lobes. The final result is displayed in Fig. 2b, featuring an approx. threefold improved axial resolution throughout the image. The arrows in Fig. $2 \mathrm{~b}$ indicate locations where the resolution improvement is prominent, especially those areas of the samples where the microtubules are so dense that they are separated only by 4Pi-confocal microscopy. In our images the pixelation is about 4-5 times denser in $x$ and $z$ direction than the correspondent width of the effective focus. This all owed us to apply an appropriate smoothing filter. To provide a feel for the data, in Fig. 2 neither the confocal nor the 4Pi data are smoothed; the pixels show the raw intensity values.

The intensity profiles in Figs. $2 d, 2 e$, and $2 f$ correspond to the axial profiles along the marked line in (a) the confocal data, (b) the 4Pi-confocal data with removed lobes, and (c) the raw 4Pi-confocal data, respectively. They represent intensity profiles through single microtubules. Hence, the curves in Figs. 2d and 2earea measure for theaxial resolution 
a

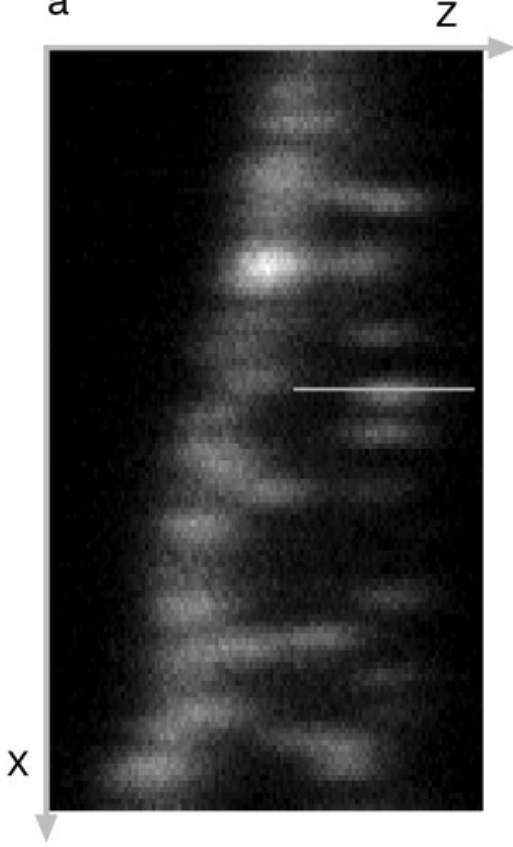

C

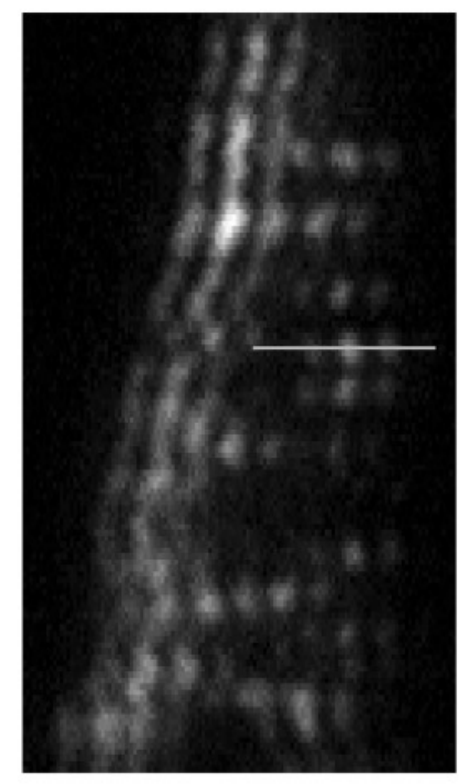

b

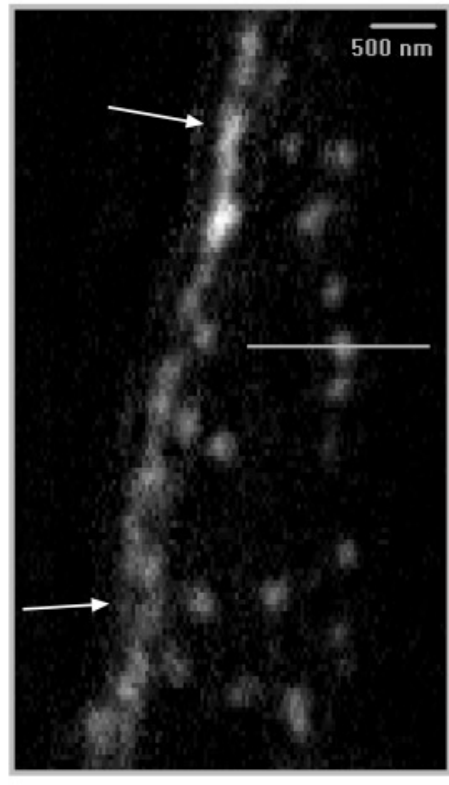

$\max$

d
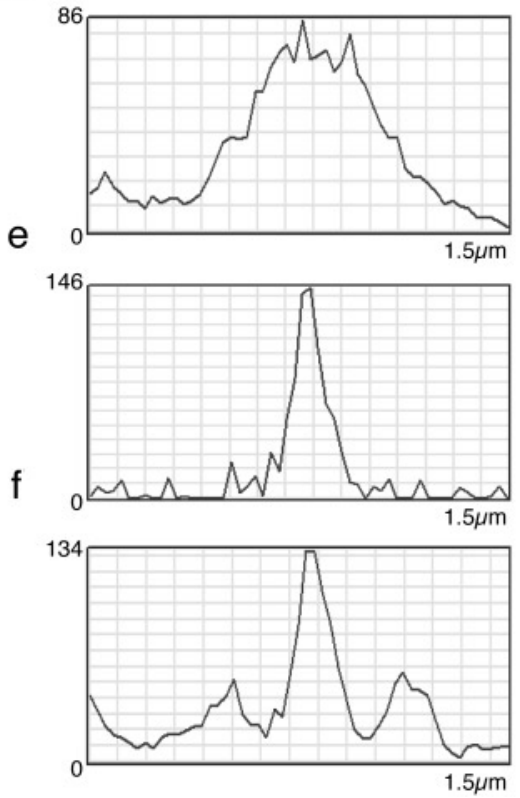

FIG. 2. (a) Confocal, (b) point-deconvolved $4 \mathrm{Pi}$-confocal, and (c) raw 4Pi-confocal xz image of microtubules in the cell. The line profiles $\mathrm{d}$-f show the response to an isolated thin fibre in the (d) confocal, (e) point-deconvolved 4Pi-confocal, and (c) raw 4Pi-confocal image. The $4 \mathrm{Pi}$-confocal response can be followed along the fibre on the left-hand side of $\mathrm{c}$. The comparison of $\mathrm{a}$ and $\mathrm{b}$ shows the increase in axial resolution as obtained with a $4 \mathrm{Pi}$-confocal microscope. The arrows in $\mathrm{b}$ indicate areas where fibres can be separated in the $4 \mathrm{Pi}$-confocal image but not in the confocal. The look-up table used in this paper is shown at the right side of b. The pixel size is 49,113, and $28 \mathrm{~nm}$ in $\mathrm{x}, \mathrm{y}$, and $z$ direction, respectively; data not smoothed.

of the 4Pi-confocal and single-lens confocal microscope, respectively.

Figures $2 b$ and $2 c$ are the fifth slice in this particular 4Pi-confocal stack ( $y=4 \times 113 \mathrm{~nm})$. We examined the adjacent $\mathrm{xz}$ slices in the stack $(y=(i-1) \times 113 n m, i=1,2,3, \ldots)$ and found that the relative phase remained constant in the following eight $x z$ images, i.e., for $1 \leq i \leq 13$. In the $x z$ slice with $i \geq 14$, however, we noticed a gradual change of the relative phase, manifested as a destructive interference where one would expect constructive interference in the image. We found that this effect occurred 
a)

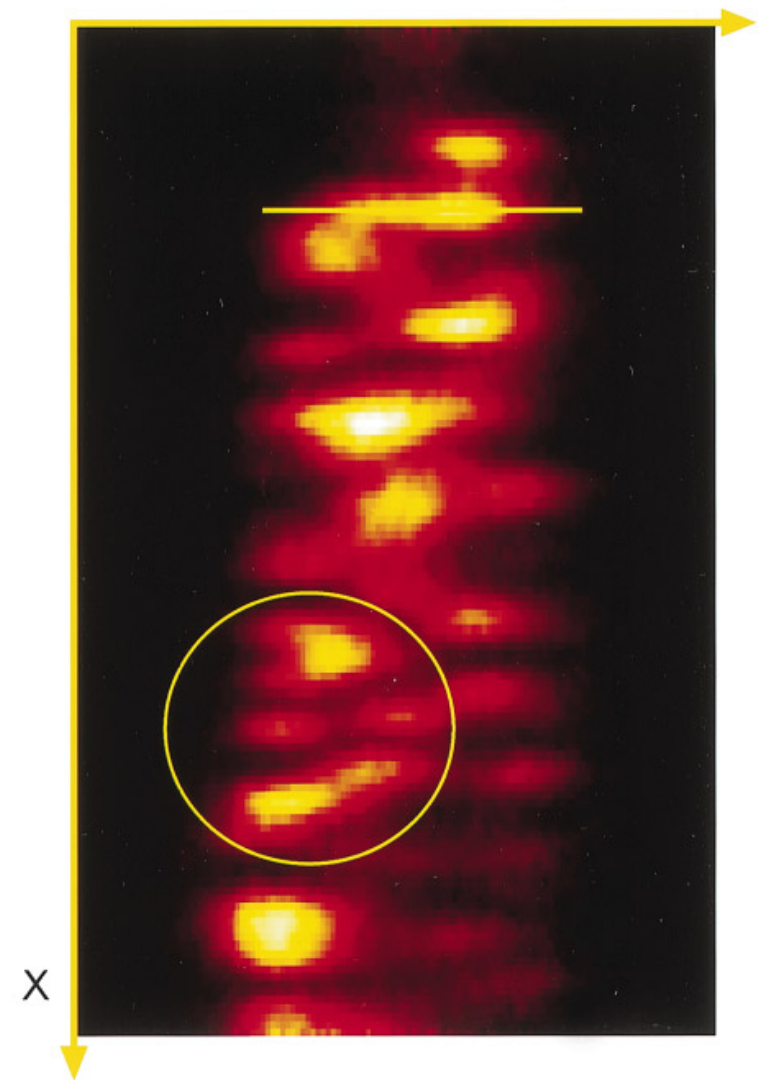

c)

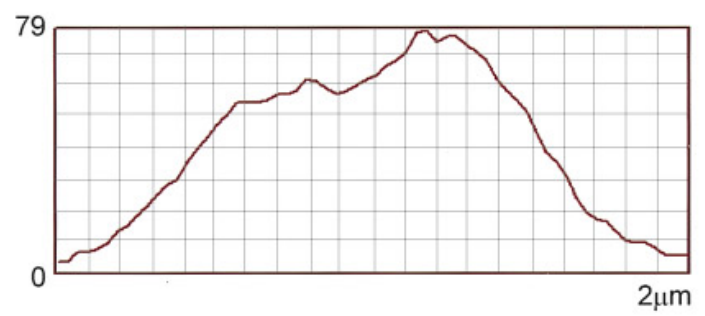

b)

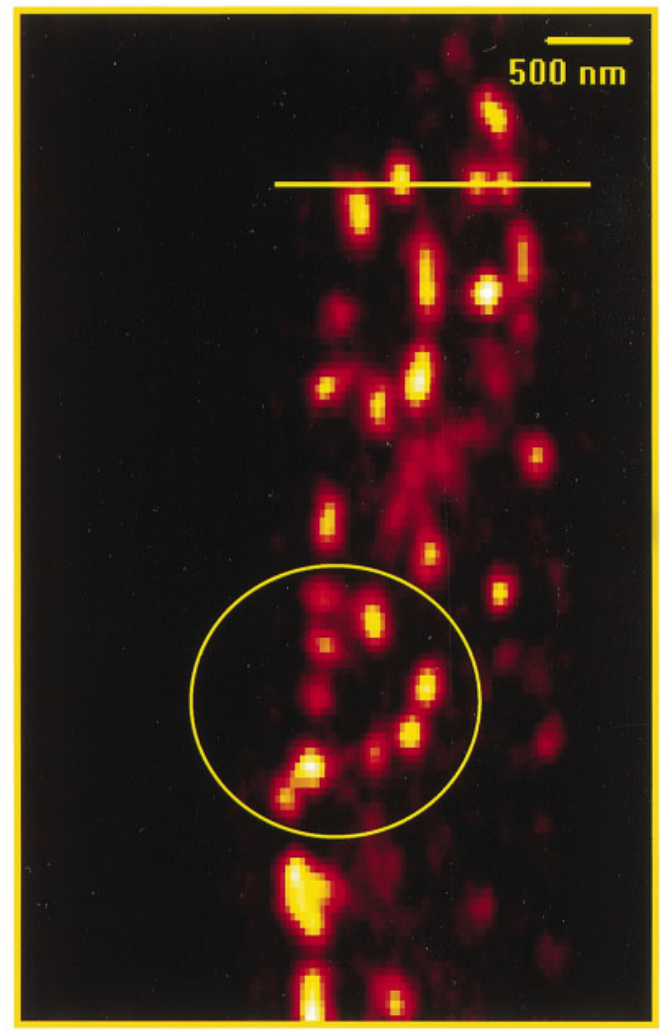

d)

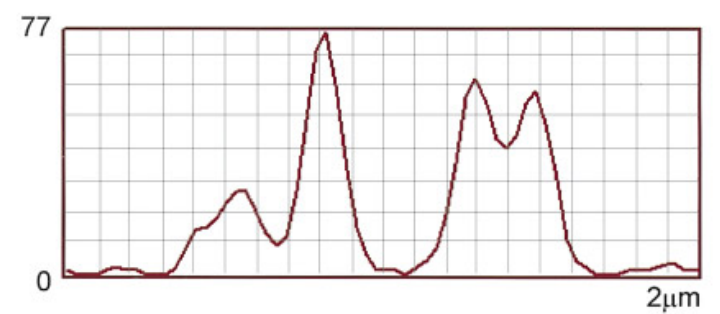

FIG. 3. (a) Confocal, (b) point-deconvolved 4Pi-confocal $x z$ images of microtubules. Curves $\mathrm{c}$ and d show a profile in the confocal and point-deconvolved $4 \mathrm{Pi}$-confocal image, along the line indicated in the images. The 4Pi-confocal image shows four fibres that are clearly separated. Note that two fibres are less than $200 \mathrm{~nm}$ apart. In this and following figures, the data is smoothed without affecting resolution.

in a region close to the cell nucleus, that is, when at least one of the focused beams seemed to propagate through the nucleus. We investigated this phenomenon by recording approximately 30 data stacks containing 8 to $64 \mathrm{xz}$ images each, totalling about $800 x z$ images. We found that with the exception of imaging certain areas affected by focusing through a medium with different index of refraction (i.e., the nucleus), the relative phase of the wavefront was largely constant. The maximum fluctuations found in the data resulted in maximal changes of the lobe height of $10 \%$. When recording the stack of which Fig. 2 is a part (total recording time of $44 \mathrm{~min}$ ), neither an alignment of the objective lenses nor an adjustment of the relative phase of the excitation beams was performed.

Figure $3 b$ shows a $x z$ slice $(i=50)$ of a stack of $\mathrm{i}_{\max }=64$ parallel $\mathrm{xz}$ images with a dimension of $128 \times 128$ pixels, along with its confocal counterpart (a). The pixel sizes in the slice are $49 \mathrm{~nm}$ in the lateral $(x)$ and $32 \mathrm{~nm}$ in the axial ( $\mathrm{z}$ ) direction. The slice distance in $\mathrm{y}$ is $70 \mathrm{~nm}$. The dense pixelation 
allowed us to apply a weighted mean filter implying the neighbouring pixels in the data set. The brightest pixel in the slice is 148 photon counts. The recording duration of one slice was $82 \mathrm{~s}$; hence, the acquisition time of the whole 3D-data stack was 88 min. The transmitted illumination beam and the image signal was monitored during image acquisition. Small corrections of alignment of the second lens were carried out after slice 18 and 35, i.e., after 25 and $48 \mathrm{~min}$, respectively. The relative phase was monitored by observing the response to single microtubules in the image. We readjusted the phase slightly at slice numbers $i=3,9,14,17,24,27,43$, $55,58,61$, and 63 . The typical phase readjustments were by typically $0.1 \pi-0.25 \pi$. No phase adjustments were carried out in the $11 \mathrm{~min}$ before and 7 min after taking the xz slice shown in Fig. 3b.

As opposed to its $\sim 550-600 \mathrm{~nm}$ value in the confocal, the effective axial resolution in the 4Pi microscope is $140-150 \mathrm{~nm}$. This improvement re sults in superior 3D imaging (Fig. 3). The upperright part of Figs. $3 \mathrm{a}$ and $\mathrm{Bb}$ and the correspondent line profile (3c and 3d) show several fibres that are well separated in the 4Pi-confocal microscope. The two fibres on the right are only $190 \mathrm{~nm}$ apart in the $z$ direction, but the intensity profile features a pronounced intensity dip of $\sim 32 \%$. In fact, the axial resolution of the $4 \mathrm{Pi}$ is better than its lateral resolution and also that of the confocal, since the typical lateral resolution is of the order of 200-230 nm for both systems.

Whereas confocal $x z$ images through the fibres appear strongly elongated along $\mathrm{z}, 4 \mathrm{Pi}$-confocal $\mathrm{Xz}$ images appear slightly narrower in z than in xy. An intuitive physical explanation for this phenomenon is that the two focused wavefronts complement each other primarily along the optic axis and therefore primarily increase the axial resolution. Because the fibres are much thinner than the effective PSF, the xz sections through thefibres represent sections through the effective focus of the microscopes; in other words they visualise the effective "diffraction pattern" of the microscope. Because the effective diffraction pattern of the 4Pi-confocal microscope is narrower, the fibres are better resolved (Figs. 3c and 3d).

The cultured cells lie flat on the coverslip so that the orientation of the microtubules is mainly parallel to the coverslip and perpendicular to the optic axis. Therefore, only an xy view of the data stack would display the fibres as lengthy, thin wires. Figure 4 shows three (Figs. 4a-4c) confocal and three (Figs. 4d-4f) 4Pi-confocal $x y$-images that are extracted from a 3D data stack consisting of 32 consecutively recorded $x z$ images. Each $x z$ image in the stack consisted of $128 \times 128$ pixels with a pixel size of 49 and $28 \mathrm{~nm}$ in $\mathrm{x}$ and $\mathrm{z}$, respectively. The lateral increment in the $y$ direction was $113 \mathrm{~nm}$. Dense pixelation below the Nyquist criterion allowed us to smooth the images as in Fig. 3. The maximum number of counts per pixel was 345 in the brightest region of the raw data. The total data acquisition time was $44 \mathrm{~min}$. Again, the relative phase and the alignment of the lenses was monitored. Neither the relative phase nor the second lens was readjusted during image acquisition.

The comparison of the xy layers in Fig. 4 again reveals the improved sectioning capability of the 4Pi-confocal microscope. The xy images are 4 pixels apart along $z$, thus amounting to $4 \times 28 \mathrm{~nm}=112$ nm increments al ong the optic axis. However, this is about 4 times less than the axial resolution of the confocal microscope. Hence, the confocal xy images (Figs. 4a-4c) show more or less the same features; all fibres appear to be in the same focal plane. In contrast, due to its fourfold improved axial resolution, the 4Pi-confocal microscope is able to better local ise and assign the fibres to different axial heights (Figs. 4d-4f).

As a next step, we investigated the potential of the $\mathrm{RL}$ restoration by applying the latter to the previous 3D stack. The effect of the 3D restoration is displayed in Fig. 5 showing a comparison between (a) the confocal, (b) point-deconvolved 4Pi-confocal, and (c) RL-restored 4Pi-confocal Xz slice of the same site of the specimen. $R L$ restoration was carried out off-line, by using the measured 3D-4Pi-confocal PSF of the microscope. The restoration was terminated after 200 iterations. The comparison of the $x z$ images shows a remarkable improvement of resolution. This is quantified in the inset figure showing theintensity profile of the data al ong the marked line.

In order to further quantify and illustrate the resolution improvement obtained by the RL-restored 4Pi-confocal microscope, we display another xz slice (Fig. 6). Adjacent microtubules with an axial distance of about 130-140 nm are featured with intensity dips of 25-40\%. Figures 5 and 6 reveal that microtubules down to a 100-nm axial distance can be distinguished in the data. This is found throughout the 3D data stack.

\section{DISCUSSION AND CONCLUSION}

Potential random changes of the relative phase of the wavefronts has initially been considered a substantial obstacle for 4Pi-confocal imaging. The approximately 800 slices we have recorded give us a feel for the applicability of the method to theimaging of microtubules in the cell. J udging from this experience we find that the relative phase of the wave fronts is largely constant in the cytsoskeleton of the 

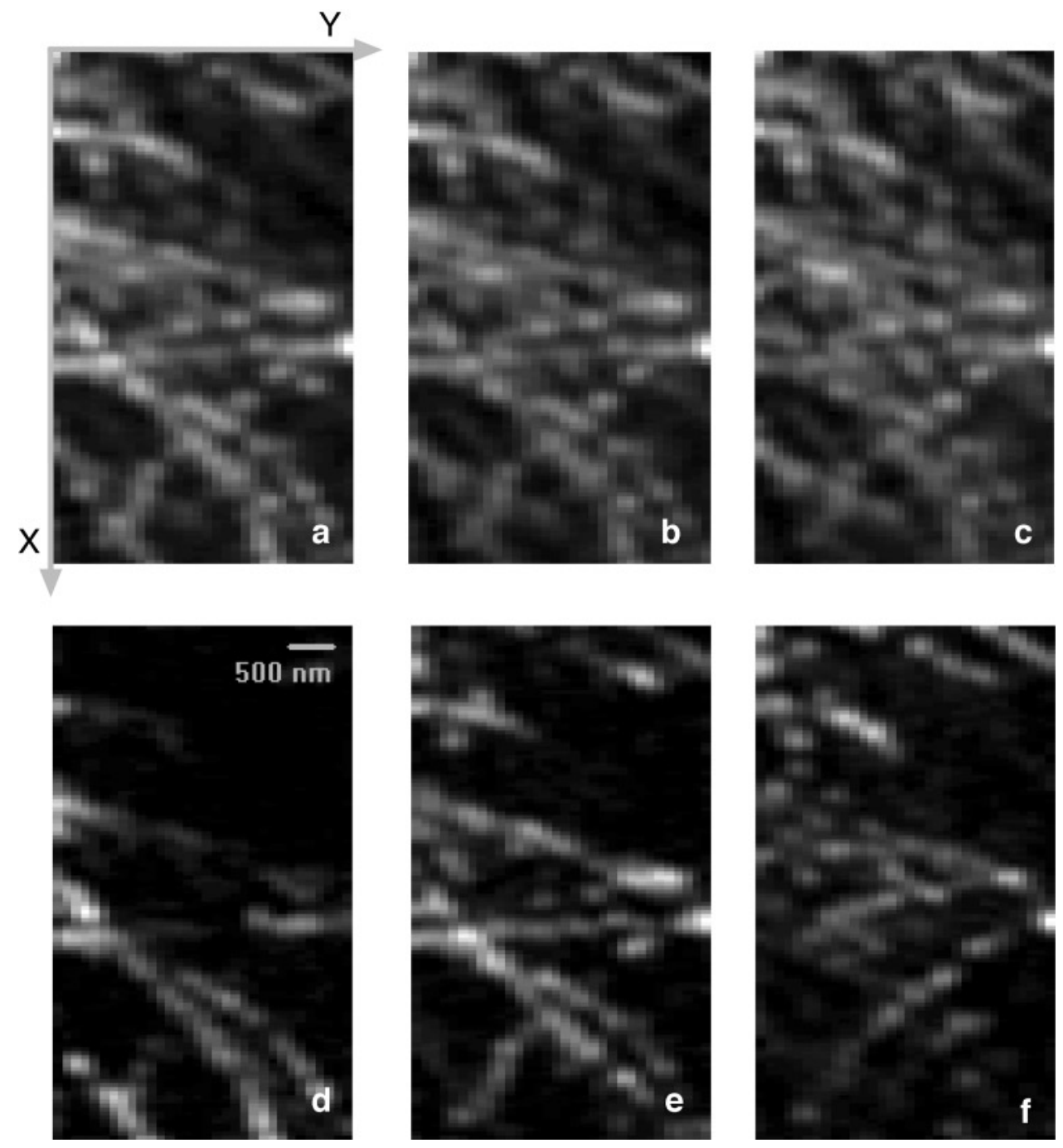

FIG. 4. Series of $x y$ images from the same part of the microtubule network of a fibroblast cell: (a-c) the confocal and (d-f) the correspondent 4Pi-confocal images. The images were arranged from a stack of 32 xz images. The xy images are $112 \mathrm{~nm}$ apart in $\mathrm{z}$. By showing different (parts of the) fibres the images $d$ - $f$ show the increased axial discrimination of the 4Pi-confocal microscope over that of the confocal.

fixed cell. As exemplified in Figs. 2, 4, 5, and 6, we could record comparatively large areas (5-7 $\mu \mathrm{m})$ without significant phase changes in the slice. The recording shown in Figs. 4- 6 was carried out without external phase or lens realignment throughout the 44 min of recording time.

In contrast, the recording in Fig. 3 required a repeated phase correction. Examination of the data stack and also the observation of the transmitted beam indicated that these areas involved focusing through a cell nucleus most likely featuring a different index of refraction. In addition, cells that partly lie on top of each other might also induce slight changes in the focused wavefronts. To be able to routinely apply $4 \mathrm{Pi}$-confocal microscopy in cellular imaging, it will be important to study potential specimen-induced phase changes in different parts of the cell, such as the nucleus, mitochondria, etc. To this end, our results demonstrate that the issue of phase change is unlikely to preclude the application of $4 \mathrm{Pi}$-confocal microscopy to cultured cells as a whole.

However, there might be cases in which 4Piconfocal microscopy will not be applicable in an unsophisticated manner. These cases are still to be determined in broad application studies but are likely to be restricted to thick and fuzzy specimens or cell nuclei. We also note that small amounts of phase noise will not so much affect the resolution as the location of the resolved object. The sharp peak of the 
a)

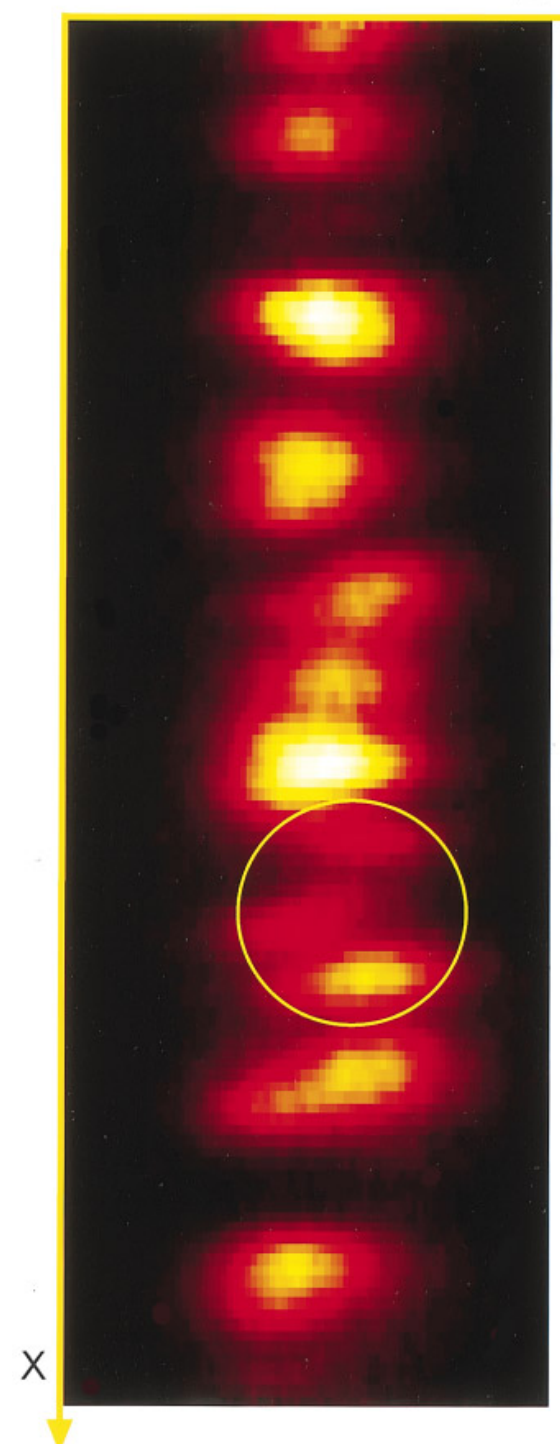

b)

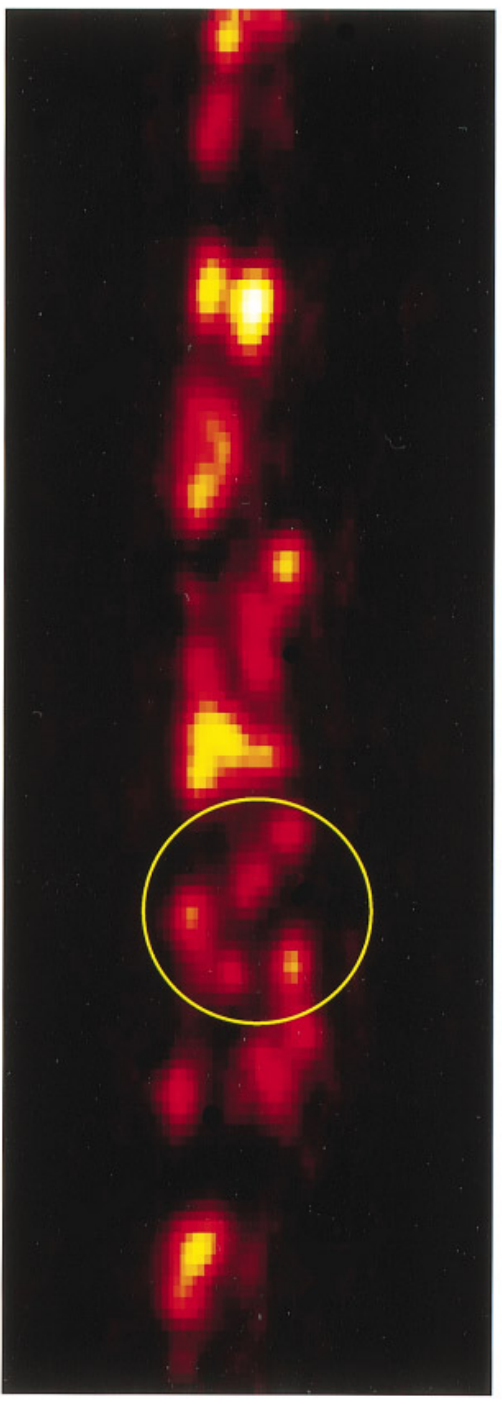

c)

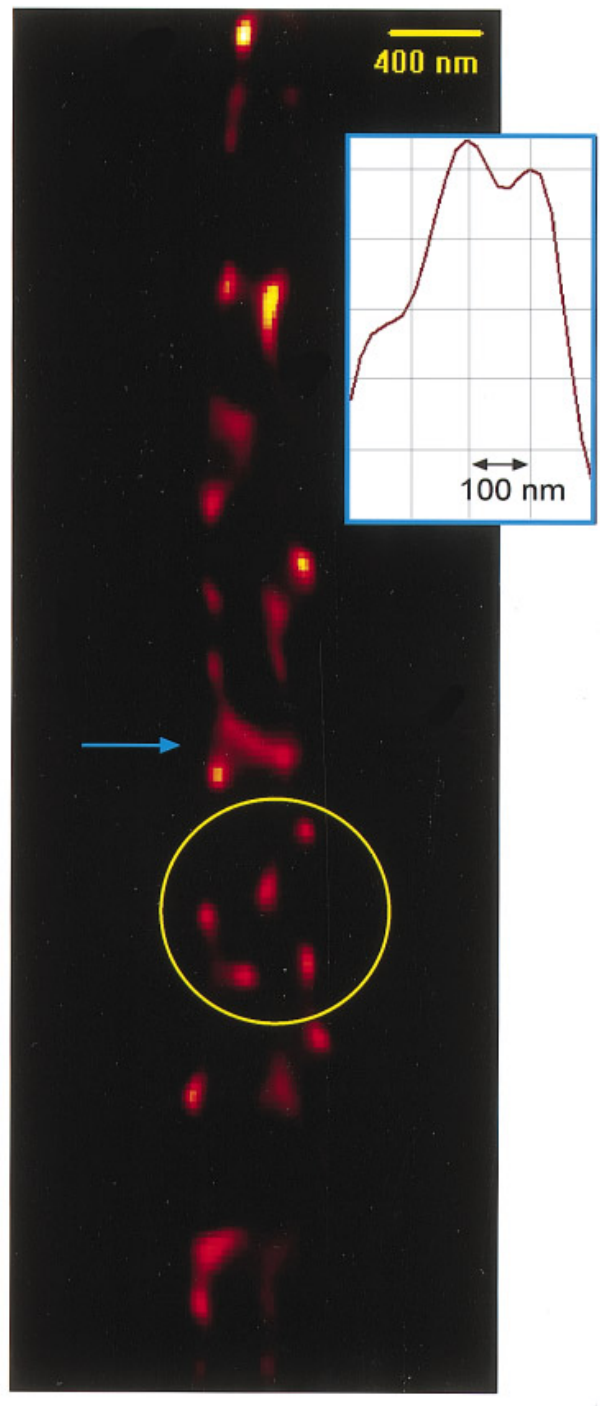

FIG. 5. (a) Confocal, (b) point-deconvolved 4Pi-confocal, and (c) restored 4Pi-confocal xz images obtained from microtubules in a mouse fibroblast cell. The images were consecutively taken at the same site of the cell. The comparison reveals a fundamental increase in $3 \mathrm{D}$ resolution in far-field fluorescence microscopy.

PSF will also be present for a slightly different relative phase, but it will be localised at a slightly offset position along z. In general, comparison with the confocal images shows that (almost) every microtubule in the 4Pi image has a counterpart in the confocal data, that is axially "smeared out," of course. Unsurprisingly the better resolution of the 4Pi images facilitated understanding of the confocal data. Wealso anticipate that object-induced phase changes will mainly restrict the size of the 4Pi images that can be obtained in a single run without readjust- ment. Another effect of phase noise is that it might affect the dynamics in the image. However, we would like to emphasise that in our experiments these aspects were not so dominant as to compromise the superior imaging with the 4Pi-confocal microscope.

The linear phase change caused by the mismatch in the refractive index between the mountant and the immersion oil is predictable because it depends on the refractive index of the mountant and also on the aperture. The phase change can be compensated by applying a phase ramp. According to our calcula- 
a

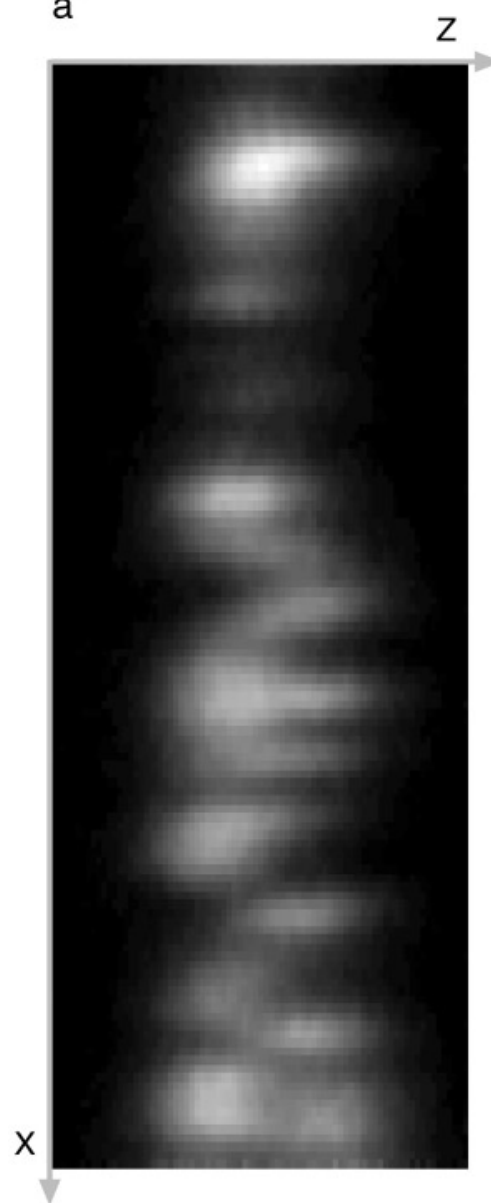

b

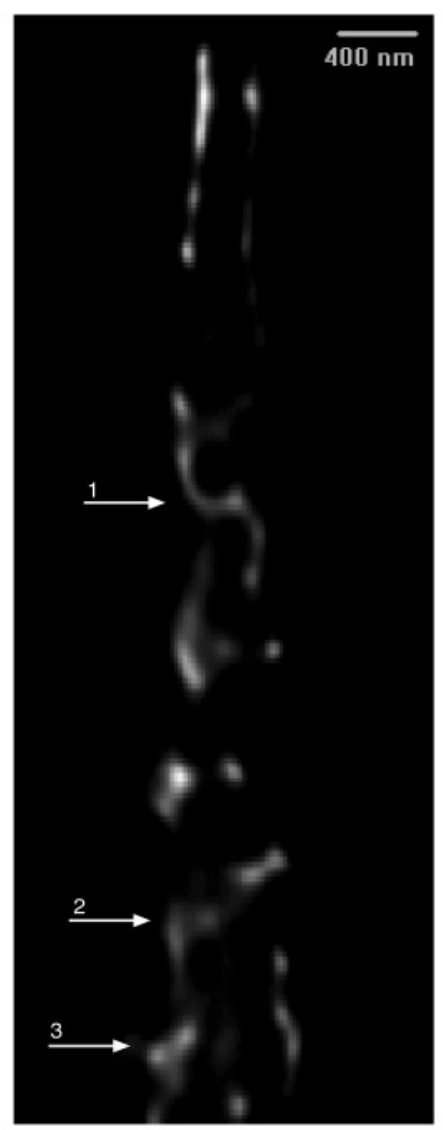

C
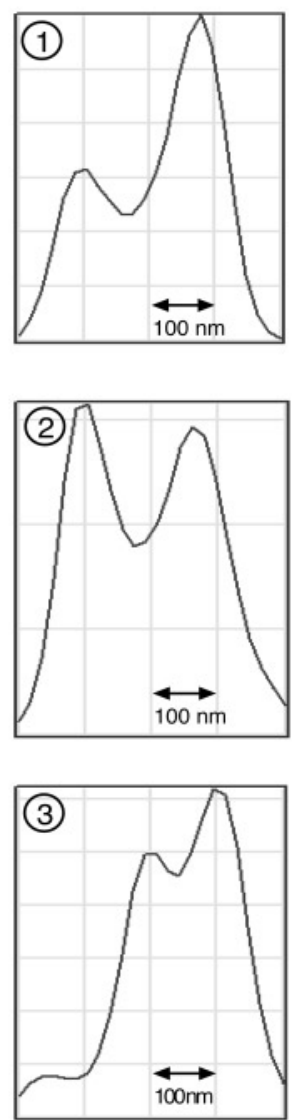

FIG. 6. (b) Restored 4Pi-confocal $x z$ images of microtubules in a cell feature an axial resolution in the 100-nm range. The normalised intensity profiles al ong the axial lines marked with 1, 2, and 3 are displayed in c. I mage a shows the confocal data taken at the same site in the cell.

tions, it should not pose difficulties for glycerol/ moviol ( $n=1.46)$ mounted samples thinner than about $20 \mu \mathrm{m}$ (Egner et al., 1998). Clearly, it is not possible to tell in advance the relative phase of the wavefronts, so it is necessary to establish (constructive) interference immediately before imaging. This problem was solved by adjusting the relative phase on a microtubule in the specimen or any other structure thinner than about $80 \mathrm{~nm}$. Alternatively, the specimen could be mounted on a coverslip that is covered with an ultrathin fluorescent layer (Schrader et al., 1998a).

The axial resolution improvement is mostly apparent in Figs. 5a-5c, showing a confocal, 4Pi-confocal, and restored $4 \mathrm{Pi}$-confocal image in a cell, as well as in Fig. 6. The resolution improvement obtained by (point-deconvolved) 4Pi-confocal microscopy is by a factor of 3-4 over the confocal along the optic axis. It amounts to $140-150 \mathrm{~nm}$ at a two-photon excitation wavelength of $\sim 800 \mathrm{~nm}$. In other words, whereas the confocal microscope produces xy slices of thickness $\Delta \mathrm{z} \approx 500-650 \mathrm{~nm}$, the $4 \mathrm{Pi}$-confocal microscope is able to produce xy slices that are effectively only $\Delta z \approx$ 140- to $150-n m$ thick.

On-linesideloberemoval by point deconvolution is highly advantageous because the final image can be immediately displayed on the monitor. We also note that on-line point deconvolution strictly requires the separability of the PSF into an axial and lateral function, as described in Eq. (1). This condition is well met in our microscope because the effective PSF resembles a function that is proportional to the cube of the focal intensity. Two orders of this dependence stem from two-photon excitation and another one stems from the detection through the confocal pinhole. Thus, side maxima and outer parts of the PSF are suppressed. Hence, the profile along $z$ does not significantly change with lateral defocus al ong $x, y$.

This condition is not fulfilled in a standing-wave microscope that uses a flat standing-wave pattern in 
a conventional epifluorescent microscope (Bailey et al., 1993). In a standing-wave microscope, the PSF is characterised by three to five side lobes on either side which gain relative strength with lateral defocus. The lobes are typically 160-200 nm apart from each other. While the standing wave microscope provides a quick overview of the sample and also a narrower FWHM, point deconvolution might not be possible. We think that in the standing-wave microscope it is not straightforward to mathematically produce a single sharp layer in the object, unless an off-line restoration is applied. The latter must rely on the full 3D PSF (Gustafsson et al., 1997). Without restoration, there is uncertainty in choice of which of the two to three almost equally bright layers actually contain the viewed object (Freimann et al., 1997).

In 4Pi-confocal microscopy, off-line restoration is simply an option that can be used to further increase the resolution, as shown in Figs. 5 and 6. Naturally, confocal data can also be improved by restoration. However, this improvement is generally less efficient than improving the resolution with a 4Pi-confocal arrangement (Hell et al., 1997) because the main improvement is due to the aperture increase.

The FWHM of the microtubules in the restored $4 \mathrm{Pi}$-confocal images is of the order of 120 and $70 \mathrm{~nm}$, in the lateral and axial direction, respectively. However, the FWHM of restored data of ultrafine structures can depend on the number of iterations in the restoration and is therefore not a decisive criterion for the resolution. A better estimate is gained through small objects that are close together but clearly distinguished in the image. The images and profiles in Figs. 5 and 6 , as well as other data we have taken, reveal an axial resolution down to $100 \mathrm{~nm}$.

We thank Dr. Donna J . Arndt-J ovin for preparing the fibroblast cells. We thank Peter J . Verveer for suggesting the convolution of the data with the object function of a band-limited fluorescent sphere to us. We also thank Christoph M. Schnetter for taking the conventional image of the fibroblast cell (Fig. 1). This work was supported by the Deutsche Forschungsgemeinschaft through a grant to S.W. H. (He-1977).

\section{REFERENCES}

Bailey, B., Farkas, D. L., Taylor, D. L., and Lanni, F. (1993) Enhancement of axial resolution in fluorescence microscopy by standing-wave excitation, Nature 366, 44-48.

Carrington, W. A., Lynch, R. M., Moore, E. D. W., Isenberg, G., Fogarty, K. E., and Fay, F. S. (1995) Superresolution in threedimensional images of fluorescence in cells with minimal light exposure, Science 268, 1483-1487.

Darnell, J., Lodish, H., and Baltimore, D. (1990) Molecular Biology of the Cell, Freeman, New York.
Denk, W., Strickler, J. H., and Webb, W. W. (1990) Two-photon laser scanning fluorescence mi croscopy, Science 248, 73-76.

Egner, A., Schrader, M., and Hell, S. W. (1998) Refractive index mismatch induced intensity and phase variations in fluorescence confocal, multiphoton and 4Pi-microscopy, Opt. Commun. 153, 211-217.

Freimann, R., Pentz, S., and Hörler, H. (1997) Development of a standing-wave fluorescence microscope with high nodal plane flatness, J. Microsc. 187, 193-200.

Goodman, J . W. (1968) Introduction to Fourier Optics, McGraw-Hill. Gu, M., and Sheppard, C. J . R. (1994) Three-dimensional transfer functions in 4Pi confocal microscopes, J. Opt. Soc. Am. A 11, 1619-1627.

Gustafsson, M. G. L., Agard, D. A., and Sedat, J. W. (1997) Sevenfold improvement of axial resolution in 3D widefield microscopy using two objective lenses, SPIE Proc. 2412, 147-156.

Hänninen, P. E., Hell, S. W., Salo, J ., Soini, E., and Cremer, C. (1995) Two-photon excitation 4pi confocal microscope: Enhanced axial resolution microscope for biological research, Appl. Phys. Lett. 66, 1698-1700.

Hell, S., and Stelzer, E. H. K. (1992a) Fundamental improvement of resolution with a 4Pi-confocal fluorescence microscope using two-photon excitation, Opt. Commun. 93, 277-282.

Hell, S., and Stelzer, E. H. K. (1992b) Properties of a 4Pi-confocal fluorescence microscope, J . Opt. Soc. Am. A 9, 2159-2166.

Hell, S. W. (1990) Double-Scanning Confocal Microscope, European Patent 0491289.

Hell, S. W. (1997) Increasing the resolution of far-field fluorescence light microscopy by point-spread-function engineering, in Lakowicz, J . R. (Ed.), Topics in Fluorescence Spectroscopy, pp. 361-422, Plenum, New York.

Hell, S. W., Lindek, S., and Stelzer, E. H. K. (1994) Enhancing the axial resolution in far-field light microscopy: Two-photon excitation 4Pi-confocal fluorescence microscopy, J. Mod. Opt. 41, 675-681.

Hell, S. W., Schrader, M., and van der Voort, H. T. M. (1997) Far-field fluorescence microscopy with three-dimensional resolution in the $100 \mathrm{~nm}$ range, J . Microsc. 185, 1-5.

Holmes, T. J ., Bhattacharyya, S., Cooper, J. A., Hanzel, D., Krishnamurthi, V., Lin, W., Roysam, B., Szarowski, D. H., and N., T. J. (1995) Light microscopic images reconstruction by maximum likelihood deconvolution, in Pawley, J . (Ed.), Handbook of Biological Confocal Microscopy, pp. 389-400, Plenum, New York.

Hulst, N. F. V., Parajo, M. F. G., Moers, M. H. P., Veerman, J . A., and Ruiter, A. G. T. (1997) Near-field fluorescence imaging of genetic material: Toward the molecular limit, J. Struct. Biol. 119, 222-231.

Kano, H., van der Voort, H. T. M., Schrader, M., van Kempen, G. M. P., and Hell, S. W. (1996) Avalanche photodiode detection with object scanning and image restoration provides 2-4 fold resolution increase in two-photon fluorescence microscopy, Bioimaging 4, 187-197.

Lindek, S., Salmon, N., Cremer, C., and Stelzer, E. H. K. (1994) Theta microscopy allows phase regulation in $4 \mathrm{Pi}(\mathrm{A})$-confocal two-photon fluorescence microscopy, Optik 98, 15-20.

Lucy, L. B. (1974) An iterative technique for the rectification of observed distributions, Astron. J . 79, 745-754.

Pawley, J . (1995) Handbook of Biological Confocal Microscopy, Plenum, New York. 
Richardson, W. H. (1972) Bayesian-based iterative method of image restoration, J. Opt. Soc. Am. 62, 55-59.

Schrader, M., and Hell, S. W. (1996) 4Pi-confocal images with axial superresolution, J . Microsc. 183, 189-193.

Schrader, M., Hofmann, U. G., and Hell, S. W. (1998a) UItrathin fluorescent layers for monitoring the axial resolution in 3Dfluorescence microscopy, J . Microsc. 190, 305-311.

Schrader, M., Voort, H. T. M. v. d., and Hell, S. W. (1998b) Three-dimensional superresolution with a 4Pi-confocal microscope using image restoration, J. Appl. Phys. 84, 1-10.
Van der Voort, H. T. M., and Strasters, K. C. (1995) Restoration of confocal images for quantitative analysis, J. Microsc. 178, 165-181.

Van Kempen, G. M. P., Van Vliet, L. J ., Verveer, P. J ., and van der Voort, H. T. M. (1997) A quantitive comparison of image restoration methods for confocal microscopy, J . Microsc. 185, 354-365.

Verveer, P., and J ovin, T. M. (1998) Image restoration based on Good's roughness penalty with application to fluorescence microscopy, J . Opt. Soc. Am. A 15, 1077-1083. 